\section{Diverse functions}

\section{D. Waterfield}

Peptide Growth Factors and Their Receptors. Volumes 1 and 2. By M. B. Sporn and A. B. Roberts. Springer: 1990. Pp.1521. DM996, \$585.80.

THE term growth factor, originally used to describe polypeptides that stimulate cell proliferation, has come to include a variety of molecules affecting growth, differentiation and development. The task of preparing Peptide Growth Factors and Their Receptors, the first major treatise on this subject, must have been daunting because of the amazing rate at which new factors and their receptors are characterized.

Progress from discovery of a factor in a tissue extract using a biological assay to definition of the sequence of the active peptide has frequently been a long and complex process which may now often be simplified with the use of recombinant DNA technology. Reviews of progress towards characterization such as found in this treatise serve to emphasize the multifunctional nature of these regulatory peptides. The process of molecular characterization has clarified and simplified the terminology introduced by researchers in their studies. The best example is found in work on the fibroblast growth factor family which now includes related peptides encoded by seven genes previously known by 22 different names and through almost as many biological assays. Thus the number of distinct factors is in a state of flux. During the time since the preparation of this treatise several novel factors have been characterized and receptors for others deduced by cloning and sequencing, a situation that is likely to go on for many years but does not detract from the current value of this excellent summary of our knowledge of growth factors up to 1990. The total number of growth-factor signal molecules remains unknown. Certainly any new treatise will be ten times the size of the present work in five years and will require some new order to be imposed on the field to help organize knowledge about such molecules.

This treatise of 1,500 pages is in two volumes which are divided into four sections. In section A there are two introductory chapters which illustrate the multifunctional nature of growth factors and the methods which have been used to isolate and characterize them. Section B contains detailed reviews of 25 distinct families of factors whose members can be grouped structurally or functionally. The content of each of these chapters falls into a pattern which reflects the gradual accumulation of a series of landmarks in knowledge of the structure-function and biological role of the factors. Epidermal growth factor leads the way, being one of the first discovered and most extensively worked on. Consequently here we can find an ency- clopaedic summary work on three-dimensional structure, mutagenesis, gene organization, receptor structure and the search for a physiological role in normal and cancer cells. Chapters devoted to platelet, insulin-like, fibroblast and transforming growth factors (TG-Fs), interleukins 1-6 (but not 7-9) and the colony stimulatory factors with erythropoietin complete volume 1 .

It is rewarding for those studying these factors to find practical clinical applications described in the chapters on colony-stimulating factors. The discovery and characterization of growth factors has lately been enormously stimulated by the interest of the biotechnology industry and there can be large rewards both in terms of beneficial clinical treatment and financial gain for those who manage to patent clinically useful factors.

The second volume deals first with the cytokines interferon and tumour necrosis factor and then with bombesin and the platelet endothelial factor. Next are reviews of factors which influence nerve cells, including nerve growth factor and a glia-derived nexin. Unfortunately the recent characterization of ciliary neurotrophic factor and brainderived neurotrophic factor is not included because it has fallen outside the time frame for preparation of this volume. Mullarian inhibiting substance which is another member of the TGF family is covered here as well as the activins and inhibins which have hormonal and growth-regulatory properties. Finally this section covers the somewhat less well known mammary-derived growth factors and the pentapeptide growth factors. Very few factors are not covered most notable perhaps being hepatocyte growth factor and leukaemia-inhibiting factor but these omissions are again due to their recent discovery.

The overall standard of the reviews in section $\mathrm{B}$ is first rate and it is wonderful to find a text which can finally be used as a reference book in this area. In some cases the detail is excessive for those not already engrossed in studies on particular factors but for those who want to read about factors in their biological context, sections $\mathrm{C}$ and $\mathrm{D}$ provide fascinating reading.

Section C includes reviews of the role of growth factors in haemopoiesis, the nervous system, cartilage and bone matabolism, the immune system, and the function of monocytes and macrophages and the extracellular matrix. Continuing in the same theme, section D reviews growth factors and proteases, inflammation and repair, angiogenesis, metastasis, development and oncogenesis. It is amazing that this monumental diversity of subject area is so well covered. This book will therefore fulfil the need for an enormous number of scientists in different disciplines to interpret and collect information on the involvement of growth factors in different areas of biology.

M. D. Waterfield is at the Ludwig Institute, 91 Ridinghouse Street, London W1P 8BT, UK.

\section{nature}

\section{is available in microform.}

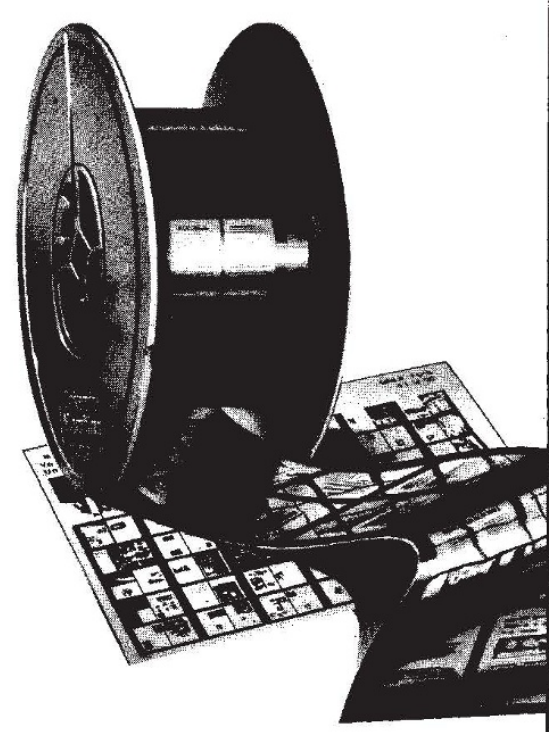

University Microfilms

International reproduces this publication in microform:

microfiche and $16 \mathrm{~mm}$ or $35 \mathrm{~mm}$ film.

For information about this publication or any of the more than 13,000 titles we offer, complete and mail the coupon to: University Microfilms International, $300 \mathrm{~N}$. Zeeb Road. Ann Arbor, MI 48106. Call us toll-free for an immediate response: 800-521-3044. Or call collect in Michigan, Alaska and Hawaii: 313-761-4700.

$\square$ Please send information about these titles:

Name

Company/Institution

Address

City

State

Phone 1

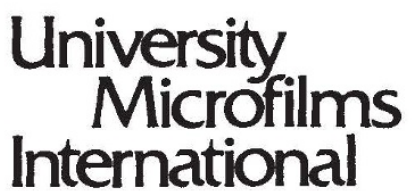

